\title{
The Transcriptional Activity of LAMP3 Gene Involved in Autophagocytosis in Colorectal Cancer LAMP3 Expression in Colorectal Cancer
}

\author{
Martyna Bednarczyk ${ }^{1}$, Małgorzata Muc-Wierzgoń1 ${ }^{1}$, Nikola Zmarzły², Celina Kruszniewska-Rajs ${ }^{2}$, \\ Beniamin Grabarek ${ }^{2}$, Agata Kaźmierczak2 , Bartłomiej Skowronek ${ }^{1}$, Klaudia Simka1, \\ Dariusz Waniczek ${ }^{3}$, Katarzyna Walkiewicz ${ }^{1}$, Urszula Mazurek ${ }^{2}$
}

${ }^{1}$ Department of Internal Medicine, School of Public Health in Bytom, Medical University of Silesia, Katowice, Poland

${ }^{2}$ Department of Molecular Biology, School of Pharmacy with the Division of Laboratory Medicine in Sosnowiec,

Medical University of Silesia, Katowice, Poland

${ }^{3}$ Chair of General, Colorectal and Polytrauma Surgery, Department of Propaedeutics Surgery, Medical University of Silesia,

Katowice, Poland

Email: martyna.bednarczyk@outlook.com

How to cite this paper: Bednarczyk, M., Muc-Wierzgoń, M., Zmarzły, N., Kruszniewska-Rajs, C., Grabarek, B., Kaźmierczak, A., Skowronek, B., Simka, K., Waniczek, D., Walkiewicz, K. and Mazurek, U. (2017) The Transcriptional Activity of LAMP3 Gene Involved in Autophagocytosis in Colorectal Cancer LAMP3 Expression in Colorectal Cancer. Journal of Biosciences and Medicines, 5, 24-36. https://doi.org/10.4236/jbm.2017.512004

Received: September 4, 2017

Accepted: November 18, 2017

Published: November 21, 2017

\begin{abstract}
Background: Autophagocytosis is a biological process involving the controlled distribution of cell fragments and organelles in order to obtain an additional source of energy. LAMP3 (lysosome-associated membrane protein 3) is a heavily glycosylated integral membrane protein located mainly in the lysosomal membrane. Recent studies have shown that it participates in tumor metastasis and drug resistance. Its main role is contribution to tumor cells proliferation, migration and invasion. The aim of this study was to determine changes in transcriptional activity of $L A M P 3$ at various stages of colorectal cancer. In addition, an attempt was made to select miRNAs potentially regulating $L A M P 3$ expression using bioinformatic databases. Methods: The study was conducted on healthy colon samples and colon cancer samples in four stages. Molecular analysis included the extraction of total RNA, purification of the obtained extracts, expression profile analysis using oligonucleotide microarray technique and in silico determination of miRNAs potentially regulating the $L A M P 3$ expression. Results: The level of $L A M P 3$ expression is higher in colorectal adenocarcinoma cells than in non-cancerous cells and depends on the stage of the disease. Conclusions: LAMP3 may promote cancer progression, metastasis and cause the resistance to treatment.
\end{abstract}

\section{Keywords}

Autophagy, Colorectal Cancer, LAMP3, Microarrays, miRNA 


\section{Introduction}

LAMP3 (lysosome-associated membrane protein 3) was discovered relatively recently by two independent researchers. De Saint-Vis initially called it DC-LAMP, which is a marker of mature dendritic cells, inducing their migration to the lymph vessels. In contrast, Ozaki described it as TSC403 with the specific expression in the lungs and overexpression in breast cancer cells. Significant similarity to the other LAMP family proteins-LAMP1 and LAMP2, was observed [1].

LAMP proteins are highly glycosylated type 1 lysosomaltransmembrane glycoproteins [1] [2]. They are located mainly in the lysosomal membrane [3] and are rarely found on the surface of normal cells, most commonly occurring in cancer cells [1] [4]. All LAMP proteins are expressed on colorectal cancer cells [4]. Recent studies suggest that LAMP3 induces differentiation of dendritic cells, which is increased in tumors of various origins. It is noted that there is a strong correlation between $L A M P 3$ expression and tumor metastases. High level of $L A M P 3$ mRNA significantly correlates with poor prognosis in patients with stage I and II tumors [4] [5] [6]. Furthermore, $L A M P 3$ takes part in the invasion and migration of cancer cells as well as the induction of autophagocytosis [7]. It also inhibits the activity of the tumor protein p53 (TP53) gene, involved in tumor metastasis and resistance to treatment. It is suggested that $L A M P 3$ may be an important molecular marker of prognosis in cancer [5].

It has long been known that hypoxia impedes the treatment of cancer. It may affect the effectiveness of radiotherapy, chemotherapy and lead to treatment resistance. In addition, it has been shown that less oxygenated tumors have a worse prognosis and higher incidence of metastasis compared with more oxygenated tumors [1] [7]. $L A M P 3$ is involved in unfolded protein response (UPR) signaling pathway induced by hypoxia or stress factors [6]. One branch of the UPR pathway involving protein kinase RNA-like endoplasmic reticulum kinase/activating transcription factor 4 (PERK/ATF4) allows cells to survive by regulating angiogenesis, apoptosis and autophagy. In addition, autophagy is believed to be a mechanism by which cancer cells overcome stressful conditions [8] [9] [10].

The PERK kinase phosphorylates the subunits of eukaryotic initiation factor $2 \alpha$ (eIF $2 \alpha$ ) in response to cellular stress, which in turn leads to translation inhibition. This regulation of translation is responsible for reducing the number of unfolded proteins in the ER. The phosphorylation of eIF $2 \alpha$ then activates the activating transcription factor 4 (ATF4), which is translocated to the nucleus, where it increases the expression of chaperone proteins (CHOP), activating transcription of the growth arrest and DNA damage-inducible protein (GADD34) and multiple proapoptotic factors as well as genes involved in amino acids metabolism, antioxidant reactions, angiogenesis and autophagocytosis [11] [12] [13].

According to the literature, among several types of autophagy LAMP3 pro- 
teins are involved in macroautophagy. However, their exact role in this process has not yet been known. It is a highly conservative process of lysosomal degradation of proteins and organelles. Autophagy is activated in response to cellular stress or nutrient deficiency [11]. Macroautophagy begins with the formation of a phagophore, it is a nucleation step where the cytoplasmic fragment along with the organelles is surrounded by a C-shaped double-membrane. Then both ends of the phagophore expand and autophagosome is formed. It undergoes a process of maturation, which causes its fusion with the lysosome to form autophagolysosome, where its contents are digested by proteolytic enzymes [14] [15].

\section{Material and Methods}

\subsection{Patients}

The study was performed in a group of 18 patients with colorectal cancer (adenocarcinoma) in various clinical stages of cancer according to the 9th Union for International Cancer Control/American Joint Committee on Cancer (UICC/AJCC) staging system. Patients were hospitalized in the Department of General Surgery at the St. Barbara Provincial Specialist Hospital in Sosnowiec. Tissue samples of colorectal adenocarcinoma and samples taken from the surgical margin of approximately $5 \mathrm{~cm}$ were collected and analyzed. The margin was assessed histopathologically and macroscopically as normal tissue. Based on histopathological analysis 18 samples were evaluated as adenocarcinoma in different clinical stages (CSI-3, CSII-5, CSIII-6 and CSIV-4 samples). The present study was approved by the Bioethical Committee of the Medical University of Silesia

(KNW/0022/KB1/42/14).

\subsection{Total RNA Extraction}

The first step was homogenization of obtained samples using a Polytron homogenizer (Kinematica, AG, Switzerland). Then, total RNA was isolated using TRIzol $^{\circledR}$ reagent (Invitrogen, Life Technologies, CA, USA) according to the attached instruction. The isolated RNA was purified using the RNeasy Mini Kit (Qiagen GmbH, Hilden, Germany) in combination with DNase I digestion. For the quantitative assessment, RNA concentration was determined using a GeneQuant II spectrophotometer, based on absorbance at $260 \mathrm{~nm}$. For qualitative evaluation, $1 \%$ agarose gel electrophoresis stained with ethidium bromide was performed.

\subsection{Microarray Analysis}

The transcriptional activity of $L A M P 3$ was determined using HG-U133A microarrays (Affymetrix, Inc., Santa Clara, CA, USA). To synthesize double-stranded cDNA 8 ug of RNA was used as a template (Super Script Choice System, Invitrogen, Life Technologies, CA, USA). The synthesis of biotinylatedc RNA was carried out using the Bioarray High Yield RNA Transcript Labeling Kit (Enzo Life Sciences, NY, USA). In order to perform fragmentation of the cRNA Sample 
Cleanup Module Kit (Qiagen GmbH, Germany) was used. Washing, staining with a complex of streptavidin-phycoerythrin and scanning the microarray in the Gene Array scanner (Agilent Technologies, CA, USA) were performed as recommended by Affymetrix Gene Expression Analysis Technical Manual.

\subsection{In Silico Identification of miRNAs}

Based on bioinformatic databases (www.microrna.org), potential miRNAs that can regulate gene expression associated with autophagy have been found. MiRanda-mirSVR algorithm, which is a combination of miRanda and mirSVR procedures, was used to search for the corresponding miRNA molecules. By this algorithm, it is possible to search for the target mRNA for the miRNA (miRanda) and to determine the correlation between them (mirSVR).

\subsection{Statistical Analysis}

Results analysis was carried out with the use of GeneSpring GX 12.6.1 software (Agilent Technologies, Inc.) and PL-Grid Infrastructure (http://www.plgrid.pl/en). In statistical analysis, the level of statistical significance $p(\alpha)<0.05$ was applied. For each of the analyzed parameters, the most important elements of descriptive statistics were determined: mean, median, minimum and maximum values, standard deviation and the upper (75\%) and lower quartiles (25\%). Differentiation of gene expression was determined by one-way analysis of variance ANOVA $(p<0.05)$. Then post hocTukey's HSD test was performed.

\section{Results}

The statistical analysis started with creating a box-and-whisker plot using the PL-Grid Infrastructure (http://www.plgrid.pl/en), which uses descriptive statistics to show the distribution of LAMP3 mRNAfluorescence signals (Figure 1, Figure 2). The control (C) and samples of colorectal cancer cells in four stages (CSI, CSII, CSIII, CSIV) are presented on the $\mathrm{x}$-axis. The values shown by box-and-whisker plot demonstrate the differences in transcriptome groups, at the level on the median, quartiles: lower-25\% and upper-75\%, and the mean value with standard deviation. Based on this graph, it can be concluded that the numerical values of fluorescence signals of selected mRNAs vary depending on the stage of cancer.

The size and statistical significance of mRNA groups differentiation were evaluated using PL-Grid program adapted to analyze Affymetrix expression microarrays. Table 1 shows the results for $L A M P 3$ gene, the directions of the observed changes in gene expression are highlighted. In the case of colorectal cancer samples, $L A M P 3$ overexpression was observed as compared to the control at the $p$ value indicating a significant strength of differentiation. For LAMP3-205569_at $p$-value is $<1.06 \times 10^{-4}$.

The results of changes in the transcriptional activity of $L A M P 3$ indicate that regardless of the cancer progression, overexpression of this gene is observed as 


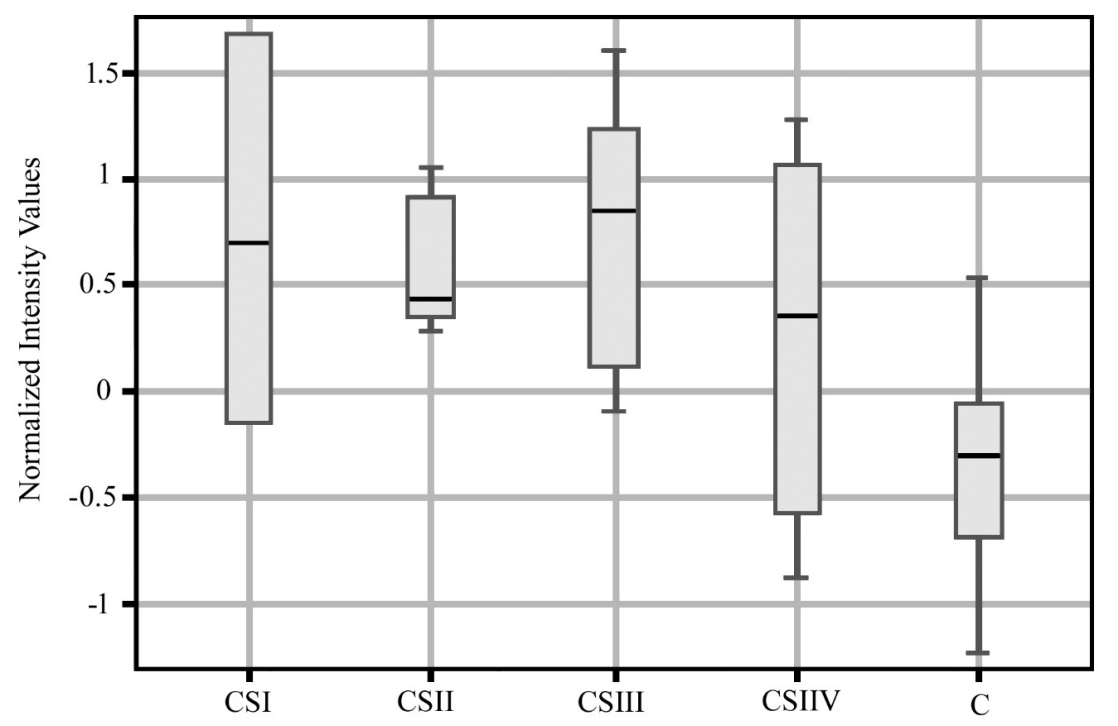

Figure 1. Descriptive statistics of the $L A M P 3$ mRNA fluorescence signals distribution (median, lower and upper quartiles). CSI, CSII, CSIII, CSIV-study groups, C-control group.

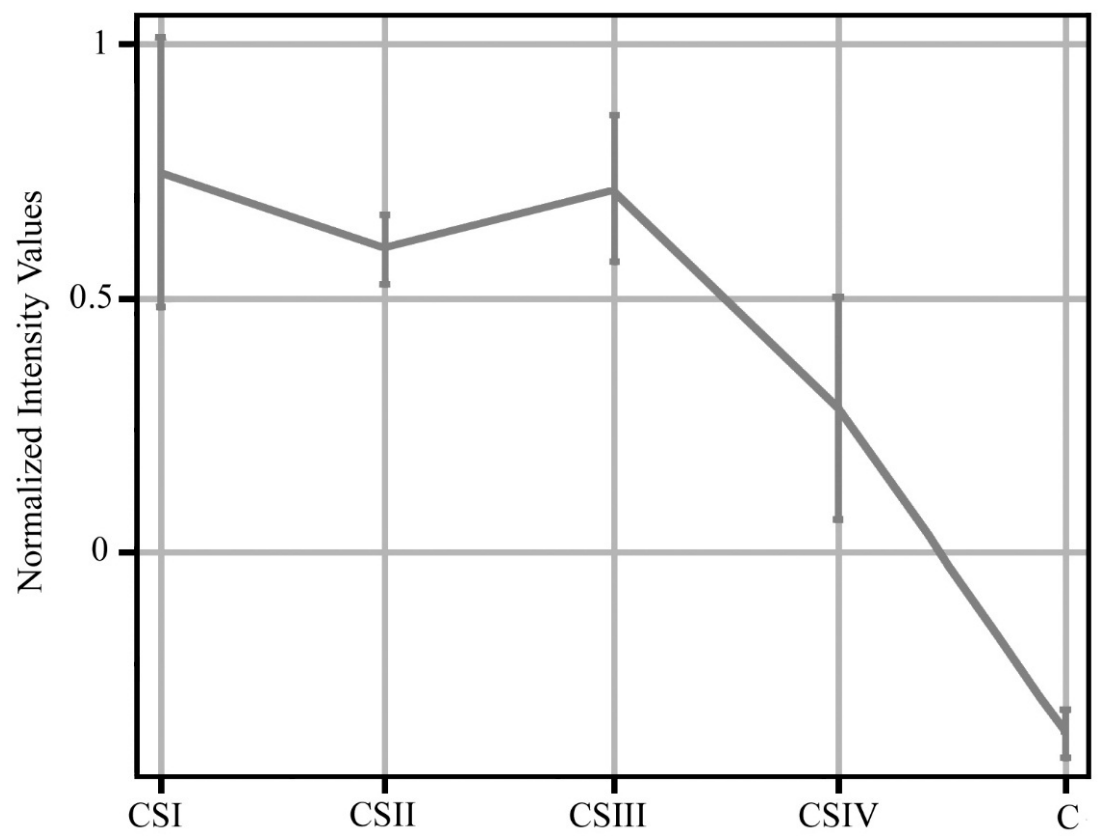

Figure 2. Descriptive statistics of the $L A M P 3$ mRNA fluorescence signals distribution (the mean value with standard deviation). CSI, CSII, CSIII, CSIV-study groups, C-control group.

Table 1. Analysis results indicating the statistical significance of $L A M P 3$ ID mRNA.

\begin{tabular}{|c|c|c|c|c|c|c|}
\hline \multicolumn{3}{|c|}{ mRNA } & \multicolumn{4}{|c|}{ FC } \\
\hline ID & Symbol & $p<0.05$ & CSI vs. C & CSII vs. C & CSIII vs. C & CSIV vs. C \\
\hline 205569_at & LAMP3 & $1.06 \times 10^{-4}$ & 2.1518712 & $1.9357514 \uparrow$ & $2.0983453 \uparrow$ & 1.5580318 \\
\hline
\end{tabular}

$\uparrow$ indicates overexpression of a gene. ID, identification number of the used probe; FC, fold-change; CSI, CSII, CSIII, CSIV, study groups; C, control group. 
compared to control. Analysis of the expression profile shows that $L A M P 3$ has the highest activity in the first stage of colorectal cancer. Based on the obtained results, it appears that the gene expression profile in CSI, CSII, CSIII stages of colorectal cancer does not undergo a relatively large variation in transcriptional activity. Large differences in the expression of $L A M P 3$ gene between the different stages of cancer samples can be observed between CSIII and CSIV, although overexpression of the analyzed transcript is still visible in comparison with the control.

The last step in the experiment was in silico determination of miRNAs potentially regulating the $L A M P 3$ expression. Taking into account the mirSVR score parameter, the cutoff of $\leq-0.70$ was applied (Figure 3 and Table 2).

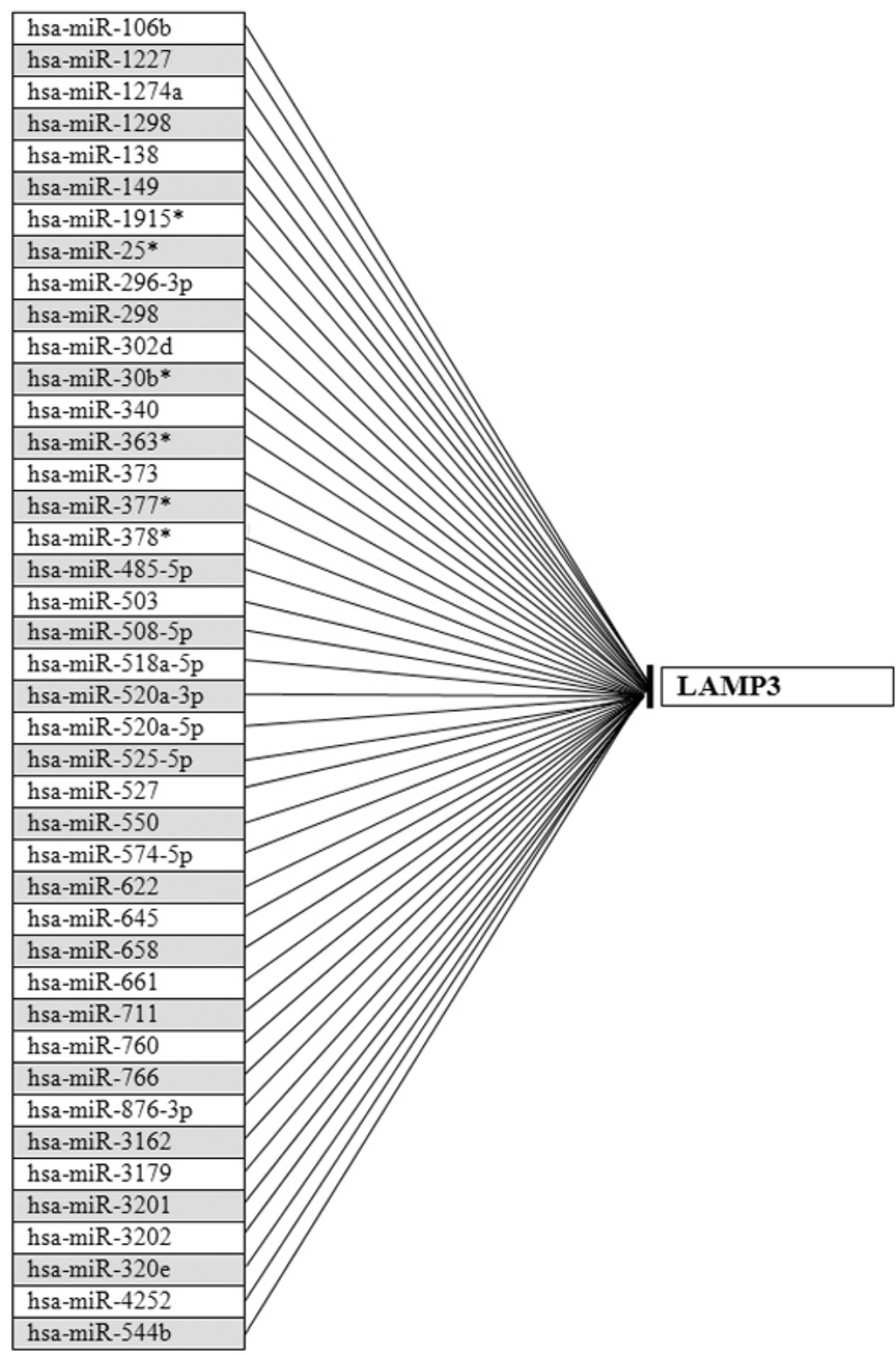

Figure 3. miRNAs potentially regulating LAMP3 expression (http://mirtar.mbc.nctu.edu.tw). 
Table 2. List of miRNAs involved in regulation of $L A M P 3$ expression (microrna.org).

\begin{tabular}{cccc}
\hline mRNA & $\begin{array}{c}\text { miRNA potentially regulating } \\
\text { expression (miRSVR score } \leq-0.70)\end{array}$ & $\begin{array}{c}\text { miRSVR } \\
\text { score }\end{array}$ & $\begin{array}{c}\text { The number of all } \\
\text { miRNAs regulating } \\
\text { mRNA expression }\end{array}$ \\
\hline hsa-miR-138 & -0.7113 & \\
hsa-miR-302d & -0.8425 & \\
hsa-miR-340* & -0.7428 & \\
hsa-miR-373 & -0.8336 & \\
haMP3 & hsa-miR-378* & -0.8318 & \\
& hsa-miR-520a-3p & -0.8300 & \\
hsa-miR-976-3p & -0.9873 & \\
hsa-miR-3201 & -1.0868 & \\
\hline
\end{tabular}

Based on the microrna.org database, it was found that 42 miRNAs may influence regulation of $L A M P 3$ expression, however 8 of them are characterized by mirSVR score $\leq-0.70$.

\section{Discussion}

This study has attempted to explain changes in the transcriptional activity of $L A M P 3$ gene involved in autophagy at various stages of the colorectal cancer. Moreover, the possibility to use this gene as an additional, complementary diagnostic marker and to determine response to treatment has been identified.

Cancer tissue samples at different stages (CSI, CSII, CSIII, CSIV) and healthy tissue samples taken from the surgical margin were examined and compared with each other. A long time of colorectal cancer formation, often the absence of characteristic precursor lesions, is the cause of a small number of samples taken from patients in stage I and II. In the early stages of the disease, there are usually no specific symptoms, which makes it difficult to collect clinical material. This study is a pilot study carried out in a small number of patients, however, we are planning to validate microarray experiment at the mRNA and protein levels. In addition, we plan to expand the study group at the moment clinical material is collected.

So far, there are very few reports on the actual role of the LAMP3 protein in the cancer progression and metastasis, but recent literature data indicate that autophagy plays a key role in these two processes. First of all, in the late stages of tumor growth, autophagy supplies the nutrients necessary for tumor cells to survive. Proteins and organelles degraded in the lysosome, provide substrates for subsequent synthesis. Mainly in the inner, poorly vascularized parts of the tumor, autophagy is a mechanism of cell survival. It also facilitates the survival of tumor cells after their detachment, as the autophagy in cells increases [16].

The results obtained in this experiment demonstrate the differentiation of $L A M P 3$ expression depending on the severity of cancer lesions of the analyzed samples. They also indicate that the $L A M P 3$ transcriptional activity differs sig- 
nificantly ( $p<0.05$ ) between healthy colorectal samples (control) and cancer tissue samples at CSI, CSII, CSIII, CSIV stages (study groups). Analysis of the $L A M P 3$ transcriptional activity shows its overexpression at each stage compared to the control, but the highest difference occurs between CSI vs. C and decreases slowly. In the first three stages of colorectal adenocarcinoma, the expression of the analyzed gene is at a similar level. Further development of cancer resulting in the classification of samples as CSIV reduces the LAMP3 copy number in comparison to previous stages. The results obtained in this experiment indicate that as the tumor progresses, the $L A M P 3$ transcription activity decreases, however, it's still overexpressed when compared to control.

$L A M P 3$ gene expression profile, in which the highest expression in the early stages of cancer decreases with its progression, may confirm the participation of protein encoded by this gene in the invasion and migration of cancer cells [7], which would be the most severe in the early stages of malignancy. Taking into account the data obtained in the experiment on the role of LAMP3 protein in promoting cancer metastasis [4] [5] [6], it is reasonable to conclude that the observations made by Kanaoet al., Liao et al., Dominguez-Bautista et al. regarding adverse prognosis in patients with stage I and II cancer are justified. In addition, according to the literature, patients with a higher level of $L A M P 3$ expression had a worse prognosis, indicating that $L A M P 3$ may be involved in the progression of colorectal cancer. Ozaki et al. carried out a study where $L A M P 3$ showed higher expression in this cancer [17].

Autophagy plays a key role in the pathogenesis of cancer. Currently accepted hypothesis states that autophagy has a double, contradictory role in the cancer development. On the one hand, it can act as a tumor suppressor, contributing to the death of cancer cells. In the early stages of the disease, autophagy has a protective effect by removing damaged proteins which prevents malignant transformation of the cells. On the other hand, it can promote oncogenesis, preventing cancer cell death and causing the resistance to treatment. It also induces tumor development, providing it with access to the nutrients, which is necessary for the metabolism and growth of cancer cells. Autophagy also affects cancer metastasis, which depends on the phase. It promotes the formation of metastases in advanced stages of the disease by increasing the survival of metastatic cells in the absence of extracellular matrix and by allowing the spread of cancer cells to distant organs [18] [19] [20] [21]. It follows that autophagy can inhibit formation of tumors or enhance it and protect cancer cells from death [22].

Autophagy is involved primarily in the regeneration of cell organelles, maintenance of energy level and protein synthesis. It is induced under conditions of cellular stress such as hypoxia or insufficient nutrients, therefore its contribution is evident in numerous diseases, including cancer [23]. In addition, many studies indicate that autophagy is a mechanism leading to cell survival under adverse conditions [24]. During the tumor progression, cancer cells are in a state of hypoxia (oxygen deficiency) due to the absence of vascularization. This condition 
contributes to the formation of cancer cells with high metastatic potential and resistance to therapy [11] [14]. Resistance develops when the partial pressure of oxygen in the tumor is less than $25-30 \mathrm{mmHg}$ [25]. In case of programmed cell death, autophagy prevents the formation of tumors. If autophagy is impaired, tumor growth by abnormal cell growth may occur [21]. Despite all the studies conducted so far, the role of autophagy in carcinogenesis is not fully understood and may be dependent on many factors, including the stage of cancer [15]. Therefore, it is reasonable to attempt to analyze the expression of genes involved in autophagy in different types of cancer and varying degrees of progression.

In our study, analysis of $L A M P 3$ transcriptional activity in colon tissue samples shows that the highest expression of this gene occurs in CSI colorectal cancer and decreases slowly, while the most visible difference in expression is observed between CSIII and CSIV. Some data indicate that in case of highly advanced cancers autophagy has the opposite effect on tumor growth. The purpose of this process could be to provide the nutrients during cancer progression. Recent studies have also shown that autophagy promotes the survival of p53-deficient cancer cells during nutrient deficiency or hypoxia. This finding indicates that inhibition of autophagy may be an effective treatment for advanced stage cancer [21].

Autophagocytosis can be induced, among other things, by UPR-dependent signaling pathway activated by hypoxia or nutrient deficiency [6] [9]. The precise mechanism of action of this pathway is not yet known, and the genes involved in this process have not yet been identified [11]. So far, it is only known that the phosphorylated transcriptional factor eIF- $2 \alpha$ activates ATF4, which results in translation inhibition and thus decrease in the amount of protein in the endoplasmic reticulum. So far, it is only known that the phosphorylated transcription factor EIF- $2 \alpha$ activates ATF4, which results in translation inhibition and thus decrease in the amount of protein in the endoplasmic reticulum [9] [26]. The role of ATF4 is, among other things, transcriptional regulation of genes responsible for amino acid metabolism and maintenance of cell homeostasis. It shows that the UPR regulates the amount of proteins at several levels, affecting the processes of transcription, translation and protein degradation. Any disturbances of the UPR pathway are part of the pathomechanism of many diseases, including cancer. It is important to know the mechanisms by which the UPR pathway contributes to the pathogenesis of these diseases. The development of new therapeutic methods requires an in-depth knowledge of the UPR mechanism and the involvement of LAMP3 in autophagy [13]. The exact meaning of the UPR signaling pathway in metastasis induced by hypoxia has not yet been investigated. It has been hypothesized that LAMP3 protein may be involved in this process because during the oxygen deficiency in tumors PERK/ATF4 pathway is activated [9]. Hypoxia in tumors is associated with insufficient oxygen supply in the tumor microenvironment. It is often connected with poor differentiation of highly advanced cancer [25]. In addition, LAMP3 is overexpressed in many types of cancers [7] [9]. 
As demonstrated by previous studies, autophagy has a crucial role in tumor development and metastasis, mainly to lymph nodes. It is believed that LAMP3 protein is involved in cancer cell resistance to therapy (chemotherapy and radiotherapy) and the mechanism of resistance involves the induction of the UPR pathway and autophagy [6]. Increased expression of ATF4 is associated with decreased sensitivity to certain types of therapy [22]. Further reports that LAMP3 participates in the dysfunction of the ubiquitin-proteasome system are also associated with drug resistance and may be important for cancer research. The resistance induced by LAMP3 is primarily directed towards proteasome inhibitors used during chemotherapy. In conclusion, it has been found that increased expression of $L A M P 3$ contributes to the development of autophagy and cell survival [6] [27]. It is important when developing new therapies, including molecularly targeted therapies, to inhibit the specific cytokine activating the signaling pathway involved in a particular disease. This type of therapy is based on the use of biological medicines, which, despite their high degree of safety, are not free from side effects [28] [29].

Despite the potential risk, it should be noted that personalized therapy (molecular targeting) is beneficial for the patient due to the ability to select pharmacotherapy based on the appropriately selected drug and its dose. The ability to more accurately predict the effects of a particular therapy on a patient will help to reduce the number of side effects that are very common in traditional therapies such as in oncology. Therefore, it is important to monitor the effectiveness of molecular markers. This would allow to select the right treatment strategy before any adverse changes occur, including the drug resistance. In order to introduce innovative therapies, it is also necessary to find new prognostic markers and to better understand the mechanism of tumor formation [7].

Colorectal cancer is one of the most commonly occurring cancers in the Western countries. Studies carried out over the last 15 years have shown that it is a multistep genetic disease, however environmental factors may promote its development [30] [31]. Very often colorectal cancer ends with death. The cause of the high mortality rate is too late diagnosis of the disease and the implementation of inadequate treatment [32]. Studies in recent years are largely focused on the molecular basis of the disease. The main objective is not only a better disease prevention and early detection, but also to learn new, effective methods to fight the disease. However, knowledge of carcinogenesis at the molecular level is still insufficient [33].

In summary, the presented study shows that the level of $L A M P 3$ expression is higher in colorectal adenocarcinoma cells than in non-cancerous cells and depends on the stage of the disease. Therefore, further studies at the molecular level are needed to help identify, among other things, predictive and diagnostic markers of this disease and the signaling pathways contributing to its development. A better understanding of the role of $L A M P 3$ in the regulation of autophagy will be important in the development of novel therapeutic agents in the near future [34]. 


\section{Acknowledgements}

This research was supported in part by PL-Grid Infrastructure.

Martyna Bednarczyk, Urszula Mazurek and Małgorzata Muc-Wierzgoń conceived and designed the experiments; Celina Kruszniewska-Rajs, Nikola Zmarzły and Agata Kaźmierczak performed the experiments; Martyna Bednarczyk analyzed the data; Martyna Bednarczyk, Katarzyna Walkiewicz and Beniamin Grabarek wrote the paper; Nikola Zmarzły and Bartłomiej Skowronek translated the paper.

\section{Conflicts}

The authors declare that there are no conflicts of interest.

\section{References}

[1] Nagelkerke, A., Mujcic, H., Bussink, J., et al. (2011) Hypoxic Regulation and Prognostic Value of LAMP3 Expression in Breast Cancer. Cancer, 117, 3670-3681. https://doi.org/10.1002/cncr.25938

[2] Grützkau, A., Smorodchenko, A., Lippert, U., et al. (2004) LAMP-1 and LAMP-2, But Not LAMP-3, Are Reliable Markers for Activation-Induced Secretion of Human Mast Cells. Cytometry, 61A, 62-68. https://doi.org/10.1002/cyto.a.20068

[3] Saftig, P., Schröder, B. and Blanz, J. (2010) Lysosomal Membrane Proteins: Life between Acid and Neutral Conditions. Biochemical Society Transactions, 38, 1420-1423. https://doi.org/10.1042/BST0381420

[4] Kanao, H., Enomoto, T., Kimura, T., et al. (2005) Overexpression of LAMP3/TSC403/DC-LAMP Promotes Metastasis in Uterine Cervical Cancer. Cancer Research, 65, 8640-8645. https://doi.org/10.1158/0008-5472.CAN-04-4112

[5] Liao, X., Chen, Y., Liu, D., et al. (20115) High Expression of LAMP3 Is a Novel Biomarker of Poor Prognosis in Patients with Esophageal Squamous Cell Carcinoma. International Journal of Molecular Sciences, 16, 17655-17667. https://doi.org/10.3390/ijms160817655

[6] Dominguez-Bautista, J.A., Klinkenberg, M., Brehm, N., et al. (2015) Loss of Lysosome-Associated Membrane Protein 3 (LAMP3) Enhances Cellular Vulnerability against Proteasomal Inhibition. European Journal of Cell Biology, 94, 148-161. https://doi.org/10.1016/j.ejcb.2015.01.003

[7] Sun, R., Wang, X., Zhu, H., et al. (2014) Prognostic Value of LAMP3 and TP53 Overexpression in Benign and Malignant Gastrointestinal Tissues. Oncotarget, 5, 12398-12409. https://doi.org/10.18632/oncotarget.2643

[8] Nagelkerke, A., Bussink, J., Mujcic, H., et al. (2013) Hypoxia Stimulates Migration of Breast Cancer Cells via the PERK/ATF4/LAMP3-Arm of the Unfolded Protein Response. Breast Cancer Research, 15, R2. https://doi.org/10.1186/bcr3373

[9] Mujcic, H., Nagelkerke, A., Rouschop, K.M., et al. (2013) Hypoxic Activation of the PERK/eIF2a Arm of the Unfolded Protein Response Promotes Metastasis through Induction of LAMP3. Clinical Cancer Research, 19, 6126-6137. https://doi.org/10.1158/1078-0432.CCR-13-0526

[10] Wang, M. and Kaufman, R. (2014) The Impact of the Endoplasmic Reticulum Protein-Folding Environment on Cancer Development. Nature Reviews Cancer, 14, 581-597. https://doi.org/10.1038/nrc3800 
[11] B'chir, W., Maurin, A.C., Carraro, V., et al. (2013) The eIF2a/ATF4 Pathway Is Essential for Stress Induced Autophagy Gene expression. Nucleic Acids Research, 41,7683-7699. https://doi.org/10.1093/nar/gkt563

[12] Blázquez, A.B., Escribano-Romero, E., Merino-Ramos, T., et al. (2014) Stress Responses in Flavivirus-Infected Cells Activation of Unfolded Protein Response and Autophagy. Frontiers in Microbiology, 5, 266.

https://doi.org/10.3389/fmicb.2014.00266

[13] Kadowski, H. and Nishitoh, H. (2013) Signaling Pathways from the Endoplasmic Reticulum and Their Roles in Disease. Genes (Basel), 4, 306-333. https://doi.org/10.3390/genes4030306

[14] Polewska, J. (2012) Autophagy-Molecular Mechanism, Apoptosis and Cancer. Postępy Higienyi Medycyny Doświadczalnej, 66, 921-936. https://doi.org/10.5604/17322693.1021109

[15] Świderek, E. and Strządała, L. (2013) Autophagy and BNIP3 Protein in Tumorogenesis. Postęy Higienyi Medycyny Doświadczalnej, 67, 363-370. https://doi.org/10.5604/17322693.1048712

[16] Dereń-Wagemann, I., Kiełbiński, M. and Kuliczkowski, K. (2013) Autofagia Autophagy-Process with Two Faces. Acta Haematologica. Polonica, 44, 383-391. https://doi.org/10.1016/j.achaem.2013.05.003

[17] Ozaki, K., Nagata, M., Suzuki, M., et al. (1998) Isolation and Characterization of a Novel Human Lung-Specific Gene Homologous to Lysosomal Membrane Glycoproteins 1 and 2: Significantly Increased Expression in Cancers of Various Tissues. Cancer Research, 58, 3499-3503.

[18] Kania, E., Pająk, B. and Orzechowski, A. (2015) Calcium Homeostasis and ER Stress in Control of Autophagy in Cancer Cells. Biomed Research International, 2015, Article ID: 352794. https://doi.org/10.1155/2015/352794

[19] Burada, F., Nicoli, E.R., Ciurea, M.E., et al. (2015) Autophagy in Colorectal Cancer: An Important Switch from Physiology to Pathology. World Journal of Gastrointestinal Oncology, 7, 271-284. https://doi.org/10.4251/wjgo.v7.i11.271

[20] Münz, Ch. (2011) Antigen Processing by Macroautophagy for MHC Presentation. Frontiers in Immunology, 2, 42. https://doi.org/10.3389/fimmu.2011.00042

[21] Eskelinen, E.L. and Saftig, P. (2009) Autophagy: A Lysosomal Degradation Pathway with a Central Role in Health and Disease. BiochimBiophysActa, 1793, 664-673. https://doi.org/10.1016/j.bbamcr.2008.07.014

[22] Badadani, M. (2012) Autophagy Mechanism, Regulation, Functions, and Disorders. Cell Biology, 2012, Article ID: 927064. https://doi.org/10.5402/2012/927064

[23] Wilke, S., Krausze, J. and Büssow, K. (2012) Crystal Structure of the Conserved Domain of the DC Lysosomal Associated Membrane Protein Implications for the Lysosomalglycocalyx. BMC Biology, 10, 62. https://doi.org/10.1186/1741-7007-10-62

[24] Nagelkerke, A., Sieuwerts, A.M., Bussink, J., et al. (2014) LAMP3 Is Involved in Tamoxifen Resistance in Breast Cancer Cells through the Modulation of Autophagy. Endocrine-Related Cancer, 21, 101-112. https://doi.org/10.1530/ERC-13-0183

[25] Qiu, X., You, Y., Huang, J., et al. (2015) LAMP3 and TP53 Overexpression Predicts Poor Outcome in Laryngeal Squamous Cell Carcinoma. International Journal of Clinical Experimental Pathology, 8, 5519-5527. https://www.ncbi.nlm.nih.gov/pmc/articles/PMC4503130

[26] Arensdorf, A.M., Diedrichs, D. and Rutkowski, D.T. (2013) Regulation of the Tran- 
scriptome by ER Stress Non-Canonical Mechanisms and Physiological Consequences. Frontiers in Genetics, 4, 256. https://doi.org/10.3389/fgene.2013.00256

[27] Kirkegaard, T. and Jäättelä, M. (2009) Lysosomal Involvement in Cell Death and Cancer. Biochimicaet Biophysica Acta, 1793, 746-754.

[28] Parcheta, P. and Kłujszo, E.H. (2014) Recurrent Erythema Nodosum and Pulmonary Lymph Node Tuberculosis in a Patient Treated for Psoriatic. Dermatology Review, 101, 390-396. https://doi.org/10.5114/dr.2014.46069

[29] De Simone, C., Caldarola, G., Maiorino, A., et al. (2016) Clinical Predictors of Nonresponse to Anti-TNF-a Agents in Psoriatic Patients: A Retrospective Study. Dermatology and Therapy, 29, 372-376. https://doi.org/10.1111/dth.12364

[30] Kheirelseid, E.A.H., Miller, N. and Kerin, M.J. (2013) Molecular Biology of Colorectal Cancer: Review of the Literature. American Journal of Physiology, 3, 72-80. https://doi.org/10.4236/ajmb.2013.32010

[31] Arnold, C.N., Goel, A., Blum, H.E. and Boland, C.R. (2005) Molecular Pathogenesis of Colorectal Cancer. Implications for Molecular Diagnosis. Cancer, 104, 2035-2047. https://doi.org/10.1002/cncr.21462

[32] Liu, L., Xu, X., Jing, L., et al. (2015) Lysosomal-Associated Protein Transmembrane 4 Beta-35 Overexpression Is a Novel Independent Prognostic Marker for Gastric Carcinoma. PLoS ONE, 10, e0118026. https://doi.org/10.1371/journal.pone.0118026

[33] Grande-Pulido, E., Riquelme-Oliveia, A., Ballesteros-Bargues, J., et al. (2011) Molecular Biology of Colorectal Cancer. In: Cidón, E.U., Ed., The Challenge of Colorectal Cancer: A Review Book, Research Signpost, Kerala, India, 35-51.

[34] Markowitz, S.D. and Bertagnolli, M.M. (2009) Molecular Basis of Colorectal Cancer. The New England Journal of Medicine, 361, 2449-2460.

https://doi.org/10.1056/NEJMra0804588 